\title{
Síndrome de Burnout en piragüistas españoles de alto nivel: prevalencia en función de variables sociodemográficas y de la práctica deportiva
}

\author{
Manuel ISORNA FOLGAR ${ }^{1}$, Ma $^{\text {a }}$ José VÁZQUEZ FIGUEIREDO ${ }^{1}$, \\ Imanol PÉREZ SABORIDO ${ }^{1}$, Antonio ALIAS GARCÍA ${ }^{2}$ \\ y Raquel VAQUERO CRISTOBAL ${ }^{3}$
}

\author{
${ }^{1}$ Universidad de Vigo, \\ ${ }^{2}$ Universidad de Almería, \\ ${ }^{3}$ Universidad Católica de Murcia
}

(Recibido el 7 de Julio de 2018; Aceptado el 10 de Diciembre de 2018)

RESUMEN: Se analizó la prevalencia del síndrome de burnout en 77 piragüistas de alto nivel entre 17 y 24 años y la relación entre las puntuaciones de burnout y sus dimensiones y algunas variables sociodemográficas (sexo y edad) y de práctica deportiva (modalidad, categoría, años de práctica y volumen de entrenamiento). Para ello, los deportistas autocumplimentaron el Athlete Burnout Questionnaire. No se han encontrado casos de burnout, aunque alrededor del $10 \%$ de los piragüistas mostraron un riesgo moderado-alto de sufrirlo, destacando el agotamiento físico/emocional. Se encontró una relación entre la edad y el nivel de agotamiento fisico/emocional, siendo menor conforme la edad aumentaba. Esta misma tendencia se observó al dividir a los piragüistas en función de su categoría, mostrando los seniors menores puntuaciones de agotamiento físico/emocional que los juniors. No hubo diferencias entre grupos al dividir a la muestra en función del resto de variables.

Palabras clave: Síndrome de burnout, deportistas, piragüistas, piragüismo, alto rendimiento

Burnout syndrome among spanish high-level paddlers:

prevalence based on sociodemographic and sport practice variables

\begin{abstract}
The present study has analyzed the prevalence of burnout syndrome in 77 high-level paddlers among 17 and 24 years-old and the relationship among burnout and its dimensions and some demographic (sex and age) and sport practice (modality, category, years of practice and training volume) variables. Athletes autocompleted the Athlete Burnout Questionnaire. There were no cases of burnout, although about 10\% of the paddlers showed moderate to high risk of suffer it, especially physical/emotional exhaustion dimension. It was found that age relation with the physical/emotional exhaustion, where the more age, the less physical/emotional exhaustion score. The same tendency was observed when paddlers was classified based on the category. Senior showed less physical/emotional exhaustion scores than junior. There were not significant differences based on the other variables.
\end{abstract}

Key words: Burnout syndrome, athletes, paddlers, canoeing, high-performance 
Correspondencia: Dr. Manuel Isorna Folgar. Dr. en Psicología por la USC. Profesor Facultad Ciencias Educación y del Deporte. Campus A Xunqueira. 36005 Pontevedra| España| Tlf: +34 98680170. E-mail: isorna.catoira@uvigo.es

\section{Introducción}

En el contexto deportivo se han llevado a cabo notables intentos por definir y cuantificar el concepto de burnout debido a su gran influencia sobre el abandono deportivo (De Francisco, Garcés de Los Fayos y Arce, 2014; Feigley, 1984; Garcés de Los Fayos, Olmedilla y Jara, 2006; Reynaga y Pando, 2005). Feigley (1984) determinó que el burnout en deportistas se caracterizaba por una pérdida progresiva de idealismo, energía y motivación, apareciendo un estado de fatiga, incremento de la irritabilidad y pérdida de entusiasmo, producido por un "trabajo" duro realizado durante demasiado tiempo en situaciones de alta presión.

El burnout deportivo es un proceso continuo que va surgiendo de una manera paulatina (Garcés de Los Fayos, Olmedilla y Jara, 2006) y que se va instaurando en el individuo hasta provocar en éste los sentimientos propios del síndrome, hasta el punto de que padecerlo puede generar en el deportista una ausencia casi completa de los motivos que le habían conducido a iniciarse y mantenerse en el deporte, y aceptar como única vía posible de solución el abandono prematuro, que es aún más llamativo cuando se da en jóvenes deportistas (Garcés de Los Fayos y Cantón, 2003; Garcés de Los Fayos y Vives, 2002). No en vano son los deportistas con mayores puntuaciones de burnout los que muestran menos optimismo y resilencia (Tutte y Reche, 2016).

En la actualidad y dentro del contexto deportivo, una de las acepciones más aceptadas, define el burnout como la presencia de un agotamiento físico y emocional (AFE) originado por unas exigencias elevadas en el entorno competitivo, una baja realización de logro personal (RSL), debido a una falta de consecución de sentimientos de éxito y de crecimiento individual en el contexto deportivo, y una devaluación del deporte (DPD) que causaría una pérdida de interés por la propia actividad (Raedeke, 1997).

Los datos de diferentes trabajos empíricos de corte descriptivo apuntan a una alta prevalencia (entre el $2,77 \%$ y el $10 \%$ ) de este trastorno en la población deportiva española (Garcés de Los Fayos, 1999; Medina y García-Ucha, 2002; Pedrosa y García-Cueto, 2014; Sánchez-Alcaráz y Gómez-Mármol, 2014; Vives y Garcés de Los Fayos, 2004). Otros trabajos a nivel internacional cifran el burnout entre el 1 y el $7 \%$, mientras que un $15 \%$ puede experimentar síntomas moderados (Gould, Dieffenbach y Moffett, 2002; Gustafsson, Kenttä y Hassmeén, 2011; Gustafsson, Kenttä, Hassmeén y Lundqvist, 2007). En función del sexo, el síndrome de burnout ha mostrado cifras ligeramente superiores en mujeres que en hombres (Gustafsson et al., 2007; Harris y Smith, 2009).

La prevalencia de burnout depende directamente de la naturaleza de la modalidad deportiva que se realice. Se ha encontrado una mayor incidencia en deportistas de alto nivel o élite y también en determinados deportes, especialmente en los de resistencia, como consecuencia de tener que realizar altos volúmenes de carga en los entrenamientos para garantizar el éxito en la competición (Price y Weiss, 2000), lo que supone una alta demanda de energía y tiempo (Garcés de Los Fayos, 1999). El estrés acumulado en los entrenamientos a lo largo de la/s temporada/s se ha sugerido que puede contribuir, al menos en parte, al desarrollo del síndrome de burnout (Gustafsson et al., 2007; Sánchez-Alcaráz y Gómez-Mármol, 2014). 
De acuerdo con esto, estudios previos han encontrado que, en deportes de altos volúmenes de entrenamiento, de alto perfeccionamiento técnico y deportes individuales de alta presión competitiva se presentan mayores niveles de burnout (Balaguer, Duda, Moreno y Crespo, 2009; Gustafsson et al., 2007, 2011; Lemyre, Hall y Roberts, 2008; Nitsch, Neumaier, Marées y Mester, 2002).

También hay otras características del entorno socio-afectivo del deportista que podrían contribuir a la aparición del burnout. En este sentido, se ha encontrado que hay diversas variables potenciales predictores del síndrome, tales como dificultades de comunicación/relación con el entrenador, un estilo de crítica centrada en el error, carencia de refuerzos positivos, monotonía en el entrenamiento, aburrimiento, discordancia entre las expectativas originales y los logros finales obtenidos, falta de apoyo del grupo de referencia o intereses mercenarios de los padres (Adie y Jowett, 2010; Garcés de los Fayos y Cantón, 2003; Gustafsson et al., 2007). El riesgo de sufrir burnout es aún mayor si a alguno de estos factores se unen características personales del deportista, tales como aislamiento, falta de habilidades deportivas, ausencia de estrategias de afrontamiento, sentimientos de estar apartado, estilo de vida externo no apropiado con el necesario para ser deportista, una personalidad ansiógena o falta de optimismo (Garcés de Los Fayos, 1999; Reynaga y Pando, 2005), que no le permitan soportar las horas de entrenamiento, superar las dificultades y el fracaso (González y Ortín, 2010). No en vano, se ha encontrado que ciertas variables de la personalidad se relacionan con determinados componentes del burnout, aspecto a tener en cuenta para la prevención en la aparición de este síndrome (Pérez-Rubio, González y Garcés de los Fayos, 2017). Otras variables que aumentan el riesgo de sufrir burnout en deportistas son el idealismo, el entusiasmo y la búsqueda del perfeccionismo (Hill, Hall, Appleton y Murray, 2010), encontrándose que son aquellos deportistas más idealistas, entusiastas y perfeccionistas los que más probabilidad tienen de sufrir burnout (Garcés de Los Fayos y Vives, 2002; Hill, Hall, Appleton y Murray, 2010).

Dentro de la población de deportistas, una de las etapas más vulnerables para sufrir este síndrome es en el periodo de formación (Appleton y Hill, 2012; Gustafsson et al., 2007). Estudios previos han encontrado que los deportistas en etapa de formación suelen ser personas idealistas motivadas hacia el logro del éxito competitivo, suponiendo un gran problema para ellos la consecución de derrotas (Garcés de Los Fayos y Vives, 2002). Ante esto, en ocasiones los deportistas jóvenes no saben cómo manejar la gran carga emocional que la competición deportiva supone. Así, en el caso de no alcanzar el éxito en la competición podrían sufrir agotamiento crónico (Reynaga y Pando, 2005). Además, el síndrome del burnout está asociado con la disminución del rendimiento deportivo (Garcés de Los Fayos et al., 2006), lo que acrecienta el problema. Un reciente estudio señala el estrés, la pérdida de control, el autoconcepto deportivo y extradeportivo como los factores directamente relacionados con el síndrome de burnout, siendo sus principales consecuencias la tensión, fatiga, irritabilidad, falta de ilusión, expectativas positivas, energía y entusiasmo hacia el deporte (García-Jarillo, De Francisco y Garcés de Los Fayos, 2016).

Concretamente, el piragüismo es un deporte en el cual los atletas realizan altos volúmenes de entrenamiento, sobre todo en el piragüismo de aguas tranquilas (Isorna, Navarro y Alacid, 2014), factor asociado a la presencia de burnout (Balaguer, Duda, Moreno y Crespo, 2009; Gustafsson et al., 2007, 2011; Lemyre, Hall y Roberts, 2008; Nitsch, Neumaier, Marées 
y Mester, 2002). A esto habría que sumar la presencia de otros factores como la necesidad de rendir pese a la poca o nula paga que reciben de sus clubes o de las instituciones, siendo esto especialmente incidente en la etapa de formación (Rascado, Boubeta, Isorna y Fernández, 2014) o el alto nivel de perfeccionamiento auto-orientado con moderados-altos niveles de ambición (Hill et al., 2010), aspectos relacionados con el riesgo de sufrir burnout. Además, los niños se inician y especializan dentro del piragüismo a edades cada vez más tempranas (Rascado et al., 2014), lo que podría acrecentar el problema. Todos estos factores podrían provocar la presencia de burnout en los piragüistas, siendo necesaria una detección temprana de la aparición de este síndrome con el fin de llevar a cabo intervenciones que eviten en abandono de los piragüistas (Rial, Marsillas, Isorna y Louro, 2013; Saies, Arribas, Cecchini, Luis-De-Cos y Otaeg, 2014).

A pesar de esto, sólo un estudio ha analizado la prevalencia de burnout en piragüistas de kayak polo y slalom de élite de diferentes edades, encontrándose puntuaciones bajas a moderadas de burnout y sus dimensiones (Hill et al., 2010). No obstante, no se han encontrado estudios que hayan analizado la presencia de burnout o alguna de sus dimensiones en piragüistas de carrera en línea, a pesar de las diferencias que hay entre estas modalidades y las otras y de la importancia que tiene dentro del panorama competitivo mundial. Además, sería recomendable identificar algunas de las variables sociodemográficas y de entrenamiento que pudieran estar asociadas al burnout en los piragüistas, con el fin de trabajar a nivel de prevención/intervención, aspectos que no se han analizado en las investigaciones previas.

Por tanto, los objetivos de la presente investigación de carácter exploratorio fueron: 1) cuantificar la prevalencia del síndrome de burnout o alguna de sus dimensiones en piragüistas españoles de carrera en línea de alto nivel; y 2) analizar la relación entre algunas variables sociodemográficas y de entrenamiento y la presencia de burnout o sus dimensiones.

\section{Material y método}

\section{Participantes}

Se realizó un estudio de carácter transversal exploratorio sobre la prevalencia de burnout en piragüistas de alto nivel españoles y sobre algunos factores asociados al burnout. La muestra fue seleccionada mediante muestreo probabilístico, polietápico estratificado por afijación proporcional y por conglomerados entre piragüistas que participaron en el campeonato de España de piragüismo de pista, celebrado en agosto de 2014 en Avilés (Asturias, España) en la categoría junior (17 a 18 años), sub 23 (entre 19 y 22 años) o senior (entre 23 y 34 años). Participaron en el presente estudio 77 piragüistas de élite nacional (aquellos que habían clasificado alguna embarcación al menos para semifinales $(\mathrm{M}=20.21, \mathrm{DE}=4.39)$ lo que supuso encuestar a un tercio de los deportistas que participaron en semifinales.

\section{Procedimiento}

El comité de Bioética Institucional aprobó el estudio. Todos los participantes fueron informados del propósito del estudio y de sus derechos como participantes en el mismo. Tras esto firmaron el pertinente consentimiento informado. En el caso de los menores de edad fueron los tutores legales quienes dieron su consentimiento para la participación en el estudio. 
Para el tratamiento de datos se siguió lo establecido en la Ley Orgánica 15/1999, de 13 de diciembre, de Protección de Datos de Carácter Personal.

A todos los encuestados se les entregó una encuesta que debían autocumplimentar, siguiendo las instrucciones de la misma de manera anónima y confidencial. Todos los componentes de la muestra fueron informados del objetivo del estudio, de la voluntariedad de la participación en la investigación, de la absoluta confidencialidad de las respuestas y manejo de los datos, y de que no había respuestas correctas o incorrectas, por lo que se les solicitó que contestaran con la máxima sinceridad y honestidad. El tiempo medio de cumplimentación fue de 12 minutos.

Todas las encuestas fueron realizadas el día de recogida de las acreditaciones, en una sala anexa a donde se repartían las mismas la cual fue adecuadamente equipada para la recogida de datos, con el fin de evitar que los resultados en la competición contaminaran sus respuestas.

\section{Instrumentos}

El cuestionario constó de dos partes. En la primera se preguntó al piragüista su edad y sexo y se recogieron algunas variables relacionadas con la práctica de piragüismo. Más concretamente se solicitó a los piragüistas que indicaran cuántos años de práctica ininterrumpida de piragüismo llevaban (menos de 2 años, entre 2 y 5 años, entre 5 y 10 años, más de 10 años), las sesiones de entrenamiento semanales que realizaban (menos de 3 días, entre 3 y 5 días, más de 5 días) y la duración de la sesión de entrenamiento (menos de 1 hora, entre $1 \mathrm{~h}$ y $1 \mathrm{~h} 30 \mathrm{~min}$, entre $1 \mathrm{~h} 30 \mathrm{~min}$ y $2 \mathrm{~h}$, más de $2 \mathrm{~h}$ ), de acuerdo con lo realizado en investigaciones previas (López, Gallegos y Isorna, 2013).

La segunda parte del cuestionario estuvo compuesta por la versión española del Athlete Burnout Questionnaire (Arce, De Francisco, Andrade, Seoane y Raedeke, 2012). Este test constó de 15 ítems, con una escala de respuesta tipo Likert con cinco niveles ( $1=$ casi nunca; $2=$ pocas veces; $3=$ algunas veces; $4=a$ menudo; $5=$ casi siempre), con las que se valoraron las tres dimensiones del burnout: 1) el AFE o sensación de extrema fatiga física y mental que se producen como consecuencia de las continua e intensas demandas del entrenamiento y la competición; 2) la RSL, la cual engloba la tendencia a evaluar la propia ejecución de forma negativa y la presencia de un autoconcepto negativo; y 3) la DPD, o desarrollo de actitudes y respuestas negativas, de insensibilidad y cinismo hacia el deporte que se practica. Los valores en cada una de las dimensiones oscilaron entre 5 y 25 . A mayor puntuación en una subescala, mayor riesgo de burnout. También se sumaron las puntuaciones de las tres dimensiones para hallar la puntuación total de burnout. Posteriormente se determinó el valor T de cada uno de los participantes siguiendo los criterios de De Francisco et al. (2014) y se categorizó a los piragüistas en función de estas puntuaciones en: (1) bajo riesgo de padecer burnout (deportistas cuya puntuación $\mathrm{T}$ era igual o menor a 50); (2) riesgo moderado (deportistas con puntuaciones $\mathrm{T}$ entre 50 y 60); (3) alto riesgo (deportistas con puntuaciones $\mathrm{T}$ entre 60 y 70), (4) con burnout (deportistas con puntuaciones $\mathrm{T}$ superiores a 70). El instrumento Athlete Burnout Questionnaire mostró para la población objeto del estudio una consistencia interna de $\alpha=.823$, .798 y .831 para la AFE, RSL y DPD, respectivamente; valores similares a los encontrados en estudios previos en adolescentes y jóvenes deportistas, en los cuales también se encontró una 
alta fiabilidad test-retest (Raedeke y Smith, 2001). De hecho, este instrumento ha sido considerado el más apropiado para medir el burnout en deportistas (Raedeke y Smith, 2001).

\section{Análisis estadístico}

Para verificar la normalidad de las variables se utilizó la prueba de Kolmogorov Smirnov, que evidenció una distribución normal de las mismas. Se realizó un análisis descriptivo de cada una de las variables con la obtención de la distribución de frecuencias. Un análisis correlacional fue llevado a cabo para analizar la relación entre la edad y las variables de entrenamiento. Además, un análisis de regresión múltiple por pasos sucesivos fue empleado para analizar la influencia de la edad y de las variables de entrenamiento sobre la puntuación total de burnout o alguna de sus dimensiones. Posteriormente se realizó una prueba t para variables independientes para analizar las diferencias en función del sexo y la modalidad de piragüismo practicada en las variables de entrenamiento y el valor de burnout o sus dimensiones y un análisis ANOVA de un factor para determinar la influencia de la categoría de piragüismo sobre las varaibles de entrenamiento, y también las diferencias en función de las variables relacionadas con el entrenamiento (años de práctica, sesiones de entrenamiento semanales y duración de la sesión) y la categoría con la puntuación de burnout o sus dimensiones. Si se encontraban diferencias significativas para el efecto principal del ANOVA se realizó una comparación por pares mediante un análisis post hoc con ajuste de TukeyKramer. El nivel de significación estadística se estableció en un valor de $p<.05$. Todos los datos fueron analizados usando el paquete estadístico SPSS versión 21,0.

\section{Resultados}

De los 77 piragüistas que participaron en el estudio, 47 eran varones (61\%) y 30 mujeres (39\%). Divididos por la categoría en la que competían, 46 eran junior (59.7\%), 13 eran sub23 $(16.9 \%)$ y 18 eran senior (23.4\%). En función de la modalidad practicada, 45 eran kayakistas (58.4\%) y 32 canoístas (41.6\%). Respecto a los años de práctica, un 2,6\% de los participantes $(n=2)$ llevaban practicando menos de 2 años, un $32.5 \%(n=25)$ entre 2 y 5 años, un $37.7 \%(n=29)$ entre 5 y 10 años y un $27.3 \%(n=21)$ más de 10 años. Al analizar el número sesiones de entrenamiento semanales, se encontró que un $9.1 \%(n=7)$ entrenaban menos de 3 días, un $16.9 \%$ $(\mathrm{n}=13)$ entre 3 y 5 días y un $74.0 \%(\mathrm{n}=57)$ más de 5 días. Sobre la duración de los entrenamientos, nadie entrenaba menos de $1 \mathrm{~h}$ por sesión, un $22,1 \%(\mathrm{n}=17)$ entre $1 \mathrm{~h}$ y $1 \mathrm{~h} 30 \mathrm{~min}$, un $40.3 \%(n=31)$ entre $1 \mathrm{~h} 30 \mathrm{~min}$ y $2 \mathrm{~h}$ y un $37.7 \%(\mathrm{n}=29)$ más de $2 \mathrm{~h}$. Las correlaciones entre la edad y las variables de entrenamiento se encuentran en la tabla 1, mientras que las diferencias en función sexo, la modalidad y la categoría de piragüismo respecto a las variables de entrenamiento

Tabla 1. Correlaciones entre la edad y las variables de entrenamiento

\begin{tabular}{ccccc}
\hline & Edad & $\begin{array}{c}\text { Años de } \\
\text { práctica }\end{array}$ & $\begin{array}{c}\text { Sesiones de } \\
\text { entrenamiento }\end{array}$ & $\begin{array}{c}\text { Duración de } \\
\text { las sesiones }\end{array}$ \\
\hline Edad & - & $\mathrm{r}=.557^{*}$ & $\mathrm{NS}$ & $\mathrm{NS}$ \\
Años de práctica & - & - & $\mathrm{NS}$ & $\mathrm{NS}$ \\
Sesiones de entrenamiento & - & - & - & $\mathrm{NS}$ \\
\hline${ }^{*} p<.05$. NS: No significativo. & & & & \\
Soc, \& Educ, 2019, Vol. 11(1) & & & &
\end{tabular}


Tabla 2. Diferencias en función del sexo, la modalidad y la categoría de piragüismo practicada en las variables de entrenamiento

\begin{tabular}{|c|c|c|c|}
\hline & Años de práctica & Sesiones de entrenamiento & Duración de las sesiones \\
\hline Hombres & 3.04 .78 & $2.79 .46^{*}$ & 3.19 .74 \\
\hline Mujeres & $2.67 \quad .88$ & $2.43 \quad 0.82$ & 3.100 .80 \\
\hline Junior & $2.54 \quad .69$ & 2.76 .60 & $3.33 \quad .79$ \\
\hline Sub23 & 3.15 .69 & $2.38 \quad .65$ & $2.92 \quad .64$ \\
\hline Senior & $3.61 \quad .78$ & $2.56 \quad .71$ & $2.89 \quad 68$ \\
\hline Kayakistas & 2.87 .79 & $2.60 \quad .65$ & 3.09 .76 \\
\hline Canoístas & $2.94 \quad .91$ & 2.72 .63 & $3.25 \quad .76$ \\
\hline
\end{tabular}

$* p<.05$ respecto a mujeres; $p<.05$ respecto a categoría junior.

Respecto al burnout y sus dimensiones, los piragüistas mostraron una puntuación media de $14.29 \pm 3.67$ en la dimensión AFE, de $12.62 \pm 4.22$ en la dimensión RSL y de $10.71 \pm 4.25$ en la dimensión DPD. La puntuación total fue de 42.16 \pm 11.00 , con una puntuación $t=40.95 \pm 10.22$. Al clasificar los valores $t$ se encontró que un $87 \%(\mathrm{n}=67)$ mostraba un bajo riesgo de sufrir burnout, un $9.1 \%(\mathrm{n}=7)$ un riesgo moderado y un $3.9 \%(\mathrm{n}=3)$ un riesgo alto. No se encontraron casos de piragüistas con burnout.

La figura 1 muestra el porcentaje de casos con bajo riesgo, riesgo moderado, alto riesgo o con AFE, RSL o DPD. Se encontró que la mayoría de los piragüistas (entre un 50 y un $80 \%$ ) tenían un bajo o moderado riesgo de sufrir AFE, RSL o DPD. No obstante, hubo un porcentaje importante de participantes que mostraron un alto riesgo de sufrir o ya sufrían AFE, RSL o DPD (entre el 20 y el 50\%); especialmente en el caso del AFE, dimensión en la cual un 38.96\% mostró alto riesgo de AFE y un $11.69 \%$ ya sufría AFE.

Al realizar el análisis de regresión lineal para predecir qué variables influían significativamente sobre el burnout o alguna de sus dimensiones, sólo se encontró que la edad actúa como variable determinante en la puntuación de AFE ( $\left.p=.013 ; \mathrm{R}^{2}=.079\right)$, siendo la ecuación resultante: $\mathrm{AFE}=19.019-0.234 *$ Edad.

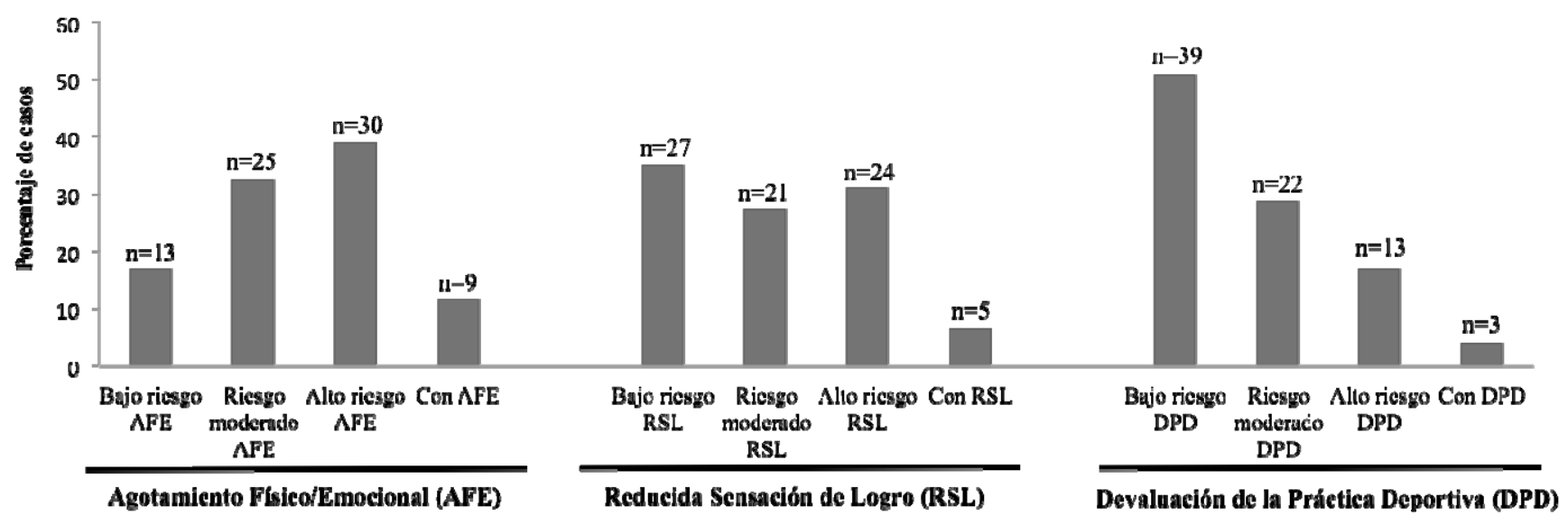

Figura 1. Porcentaje de casos con bajo, moderado o alto riesgo o con Agotamiento Físico/Emocional (AFE), Reducida Sensación de Logro (RSL) o Devaluación de la Práctica Deportiva (DPD). 
Los valores descriptivos de la puntuación total del burnout y sus dimensiones en función de las variables sociodemográficas y de entrenamiento se encuentran en la tabla 3. No se encontraron diferencias en ninguna de las variables analizadas entre sexos (puntuación total de burnout: $t(75)=.41, p=.685$; AFE: $t(75)=-.60, p=.551$; RSL: $t(75)=.22, p=.227$; DPD: $t(75)=.30$, $p=.768$ ). Tampoco cuando el análisis se hizo según la modalidad de piragüismo practicada (puntuación total de burnout: $t(75)=.96, p=.341$; AFE: $t(75)=.26, p=.796$; RSL: $t(75)=1,50$, $p=.138$; DPD: $t(75)=.317, p=.752)$.

Tabla 3. Valor medio de burnout y sus dimensiones en función de las variables sociodemográficas y de entrenamiento

\begin{tabular}{cccccc}
\hline & & $\begin{array}{c}\text { Puntuación } \\
\text { total burnout }\end{array}$ & AFE & RSL & DPD \\
\hline Sexo & Hombre & $42.57 \pm 10.36$ & $14.09 \pm 3.43$ & $13.08 \pm 4.13$ & $10.83 \pm 4.19$ \\
Categoría & Mujer & $41.52 \pm 12.08$ & $14.60 \pm 4.05$ & $11.89 \pm 4.33$ & $10.53 \pm 4.41$ \\
& Junior & $42.56 \pm 10.30$ & $14.72 \pm 2.99$ & $12.93 \pm 4.69$ & $10.22 \pm 3.75$ \\
& Sub23 & $45.15 \pm 14.17$ & $15.69 \pm 5.30$ & $13.08 \pm 4.02$ & $11.31 \pm 5.10$ \\
Modalidad & Senior & $38.98 \pm 9.99$ & $12.17 \pm 3.13$ & $11.48 \pm 2.85$ & $11.56 \pm 4.84$ \\
& Kayak & $43.18 \pm 11.49$ & $14.38 \pm 3.78$ & $13.22 \pm 4.56$ & $10.84 \pm 4.23$ \\
Años de & Canoa & $40.74 \pm 10.28$ & $14.16 \pm 3.56$ & $11.77 \pm 3.59$ & $10.53 \pm 4.34$ \\
práctica & Menos de 2 & $38.00 \pm 9.90$ & $14.00 \pm 2.83$ & $12.50 \pm 3.53$ & $7.00 \pm 1.41$ \\
& Entre 2 y 5 & $41.32 \pm 10.07$ & $14.68 \pm 3.01$ & $12.40 \pm 4.38$ & $9.56 \pm 3.78$ \\
& Entre 5 y 10 & $45.36 \pm 11.66$ & $15.07 \pm 3.91$ & $13.74 \pm 4.64$ & $11.69 \pm 4.29$ \\
Días de & Más de 10 & $39.16 \pm 10.77$ & $12.76 \pm 3.86$ & $11.35 \pm 3.19$ & $11.10 \pm 4.59$ \\
entrenamiento & Menos de 3 & $43.48 \pm 7.86$ & $13.29 \pm 2.75$ & $13.33 \pm 3.85$ & $12.57 \pm 4.68$ \\
por semana & Entre 3 y 5 & $42.36 \pm 14.71$ & $14.15 \pm 5.56$ & $12.43 \pm 4.64$ & $11.23 \pm 5.20$ \\
& Más de 5 & $41.96 \pm 10.53$ & $14.44 \pm 3.26$ & $12.57 \pm 4.23$ & $10.37 \pm 3.97$ \\
Duración de la & Entre 1h y 1h 30min & $43.78 \pm 11.93$ & $13.94 \pm 3.91$ & $13.43 \pm 4.73$ & $11.65 \pm 3.92$ \\
sesión de & Entre 1h 30min y 2h & $41.29 \pm 10.97$ & $14.26 \pm 4.13$ & $12.58 \pm 4.10$ & $10.00 \pm 4.42$ \\
entrenamiento & Más de 2h & $42.15 \pm 10.75$ & $14.52 \pm 3.05$ & $12.18 \pm 4.11$ & $10.93 \pm 4.27$ \\
\hline
\end{tabular}

AFE: Agotamiento Físico/Emocional; RSL: Reducida Sensación de Logro; DPD: Devaluación de la Práctica Deportiva

Sí que se encontraron diferencias significativas en algunas dimensiones al clasificar a los piragüistas según su categoría, hallándose diferencias significativas en la AFE $(F(74,2)=4.69, \mathrm{gl}=74(2), p=.012)$. El posterior ajuste de Tukey-Kramer mostró que los piragüistas de categoría junior tenían puntuaciones significativamente mayores que los seniors $(p<.028)$. No obstante, no se hallaron diferencias significativas en el resto de dimensiones (RSL: $F(74,2)=.86, p=.429$ y DPD: $F(74,2)=.79, p=.458)$, ni en la puntuación total de burnout $(F(74,2)=1.27, p=.286)$.

El ANOVA no mostró un efecto significativo de los años de práctica sobre el burnout $(F(73,3)=1.51, p=.219)$ y sus dimensiones (AFE: $F(73,3)=1.81, p=.154$; RSL: $F(73,3)=1.35$, (C) Psy, Soc, \& Educ, 2019, Vol. 11(1) 
$p=.264$; DPD: $F(73,3)=1.74, p=.167)$. Tampoco los días de entrenamiento semanales tuvieron un efecto significativo sobre la puntuación total de burnout $(F(74,2)=.06, p=.942)$ y sus dimensiones (AFE: $F(74,2)=.31, p=.733$; RSL: $F(74,2)=.11, p=.893$; DPD: $F(74,2)=.95$; $p=.391$ ). Lo mismo sucedió respecto al tiempo de entrenamiento diario (puntuación total de burnout: $F(74,2)=.88, p=.418$; AFE: $F(74,2)=.13, p=.878$; RSL: $F(74,2)=.46, p=.630$; DPD: $F(74,2)=.88, p=.418)$.

\section{Discusión}

El objetivo principal de la presente investigación fue analizar la prevalencia de burnout en piragüistas españoles de alto nivel. En el presente estudio se encontró que 9 de cada 10 piragüistas tenían un bajo riesgo de burnout; mientras que alrededor de $10 \%$ tenía un riesgo moderado o alto de sufrir este síndrome. No se detectaron casos de burnout, lo que viene a indicar que los piragüistas perciben su actividad deportiva con más beneficios que costos. Estos resultados podrían estar condicionados por el hecho de que la mayoría de los deportistas que abandonan la práctica de una modalidad deportiva como consecuencia del burnout lo hacen durante el periodo de iniciación, siendo los deportistas que mejor gestionan el estrés los que persisten (Vives y Garcés de Los Fayos, 2004). De hecho, se ha apuntado a que este síndrome se podría apreciar en los deportistas desde los 10 años, sobre todos en aquellos que sufren una mayor presión por presuponérseles unas habilidades que les podrían conducir al deporte de élite (García-Parra, González y Garcés de los Fayos, 2016). Los participantes del presente estudio llevaban en muchos casos más de cinco y diez años practicando piragüismo, lo que podría explicar los bajos índices de burnout hallados.

Los resultados coinciden con los encontrados en piragüistas de kayak polo y slalom de élite nacional (Hill et al., 2010). No obstante, deportistas de otras modalidades deportivas han mostrado una prevalencia de burnout y valores en las tres dimensiones superiores a los encontrados en el presente estudio (Garcés de Los Fayos, 1999; Gould et al., 2002; Gustafsson et al., 2007, 2011; Medina y García-Ucha, 2002; Pedrosa y García-Cueto, 2014; SánchezAlcaráz y Gómez-Mármol, 2014; Vives y Garcés de Los Fayos, 2004). Se ha de tener en cuenta que la muestra del presente estudio eran deportistas de élite nacional, por lo que, a pesar de las demandas de altas cargas de entrenamiento que realizan los piragüistas (Isorna et al., 2014), podrían tener una alta satisfacción como consecuencia de los resultados obtenidos, la percepción de competencia añadida, las adaptaciones en los aspectos psicológicos, así como la presencia de factores de protección personológicos propios, tal y como se han encontrado en otros deportistas (Garcés de Los Fayos y Cantón, 2003). Además, los palistas se encontraban en el momento probablemente más importante de la temporada (campeonato de España), lo que podría condicionar la percepción de burnout, tal y como han demostrado estudios previos que muestran como la manifestación e intensidad del burnout fluctúa a lo largo de la temporada (Tutte, Blasco y Cruz, 2006).

No obstante, si bien es cierto que para que un deportista presente el síndrome es necesario que se conjuguen las tres dimensiones del burnout, en la presente investigación se ha encontrado que existe un gran número de piragüistas que presenta puntuaciones altas en al menos una de ellas, lo que aumenta la vulnerabilidad a sufrir el síndrome en el futuro. Analizando las dimensiones de burnout por separado, hay que tener en cuenta que alrededor del 
$40 \%$ de los piragüistas encuestados muestra un alto riesgo de sufrir agotamiento físico/emocional, siendo ésta la dimensión que mayores puntuaciones de burnout alcanza. El piragüismo es un deporte que se caracteriza por unas duras condiciones de entrenamiento y competición influidas por las condiciones climatológicas, muchas veces poco favorables sobre todo durante los meses de invierno (Lenz, 2008) y unas altas cargas de entrenamiento (Isorna et al., 2014). Al respecto, en el presente estudio casi la totalidad de la muestra indicó entrenar más de 1 hora y media más de tres días a la semana, entrenando tres de cada cuatro piragüistas más de 5 días por semana.

Las altas puntuaciones mostradas por los piragüistas en la dimensión de agotamiento físico/emocional coinciden con lo encontrado por Hill et al. (2010) en piragüistas de slalom y de kayak polo, por Vives y Garcés de Los Fayos (2004) en un grupo de tenistas, futbolistas, atletas, ciclistas, jugadores de baloncesto y nadadores de élite y por Sánchez-Alcaráz y GómezMármol (2014) en tenistas de alto rendimiento. Sin embargo, los resultados contrastan con los hallados por De Francisco et al. (2014) en deportistas amateur de deportes colectivos en los que las mayores puntuaciones fueron obtenidas en la dimensión de devaluación de la práctica deportiva. Esto podría deberse a que el alto riesgo de agotamiento físico/emocional mostrado por los piragüistas del presente estudio y los deportistas de élite de los estudios previos (Hill et al., 2010; Sánchez-Alcaráz y Gómez-Mármol, 2014; Vives y Garcés de Los Fayos, 2004) podría ser consecuencia de las altas cargas de entrenamiento (Garcés de los Fayos y Cantón, 2003), las cuales suelen ser mayores en los deportistas de élite que en los amateur (Pedrosa y García-Cueto, 2012), o de la búsqueda de altos estándares de perfeccionamiento (Hill et al., 2010), variables que además condicionan más el rendimiento dentro de los deportes individuales que de los colectivos (Gustafsson et al., 2007).

Estudios previos han señalado que el burnout es desencadenado en el deporte de alto rendimiento por las exigencias físicas y psicológicas. Sin embargo, el burnout no es una consecuencia irremediable del alto rendimiento (Appleton y Hill, 2012), tal y cómo se ha podido comprobar en el presente estudio. Por lo tanto, la identificación de las variables psicosociales que pueden prevenir el burnout es un tema que necesita ser investigado en profundidad. En este sentido, otro de los objetivos de la presente investigación fue determinar qué variables sociodemográficas y de entrenamiento podrían estar contribuyendo a la aparición de burnout o alguna de sus dimensiones.

En el presente estudio se encontró una relación entre la edad y el agotamiento físico/emocional, encontrándose menores puntuaciones a mayor edad de los piragüistas. Ya que la edad de los piragüistas correlacionó positivamente con los años de práctica ininterrumpida de piragüismo, estos resultados podrían estar relacionados con el hecho de que es en los primeros años de práctica en los que los piragüistas muestran mayores puntuaciones de burnout y sus dimensiones (Vives y Garcés de Los Fayos, 2004). Además, los deportistas tienen que manejar una variedad de funciones en otros dominios de su vida además del deportivo, y la edad, unido a la mayor cantidad de experiencias personales, contribuye a desarrollar estrategias de afrontamiento eficientes en un intento de resolver los conflictos en los dominios de su vida, las cuales son extrapolables al ámbito deportivo y podrían disminuir las puntuaciones de burnout (Hoar, Kowalski, Gaudreau y Crocker, 2006).

No se ha encontrado ninguna variable que influya significativamente sobre la puntuación total de burnout o las otras dimensiones que lo componen. Hay que tener en cuenta (c) Psy, Soc, \& Educ, 2019, Vol. 11(1) 
que las puntuaciones en estos parámetros de los piragüistas fueron en general muy bajas y homogéneas. Por lo tanto, al no darse prácticamente el fenómeno, podría no haber variables que los determinaran estadísticamente.

Al analizar las puntuaciones de burnout o sus dimensiones dividiendo a la muestra en función de sus características sociodemográficas y deportivas, no se han encontrado diferencias estadísticamente significativas entre hombres y mujeres. Estos resultados contrastan con los mostrados por Gustafsson et al. (2007), Harris y Smith (2009) o Pedrosa y García-Cueto (2014) quienes encontraron mayores niveles de burnout en las mujeres, argumentando como principales motivos el conflicto de rol, las cargas familiares, una menor remuneración en la élite y una peor percepción del esquema corporal. Una posible explicación a los resultados detectados en el presente estudio puede ser que las mujeres de la muestra entrenaban menos días que los hombres, lo que sin lugar a duda es un factor protector del burnout (Carfagno y Hendrix, 2014). No obstante, las características de la muestra utilizada y el porcentaje de mujeres piragüistas con el que cuenta la investigación obligan a tomar estos datos con precaución.

Tampoco se encontraron diferencias significativas entre los kayakistas y canoístas en la puntuación total de burnout o alguna de sus dimensiones. Esto podría deberse a que los piragüistas de ambas modalidades realizan los entrenamientos con frecuencias y duraciones similares, con la misma metodología del entrenamiento y dinámica de grupo y en las mismas condiciones ambientales (Isorna et al., 2014).

En línea con lo hallado sobre las variables que influyen significativamente sobre el burnout o sus dimensiones, en el presente estudio se encontró que los piragüistas de categoría junior tenían mayores puntuaciones de agotamiento físico/emocional que los seniors. Esto podría deberse a que llevan más años de práctica, variable que protege la aparición de burnout y el posterior abandono deportivo (Vives y Garcés de Los Fayos, 2004) y a que pueden contar con un mayor bagaje de técnicas de afrontamiento de los problemas o situaciones que se dan en el ámbito deportivo (Hoar et al., 2006).

Por otra parte, al contrario de lo que se podría presuponerse, no se han encontrado diferencias en las puntuaciones de burnout o sus dimensiones cuando se clasificó a los piragüistas según los años de práctica que llevaban, los días de entrenamiento semanales y las horas de entrenamiento por día. Esto podría deberse a la gran homogeneidad de los resultados encontrados en estas variables.

La principal limitación del presente estudio es que sólo se incluyeron piragüistas de élite nacional. Aunque el porcentaje de participantes fue alto, estos datos no son extrapolables a deportistas de otros niveles de competición. Además, las mediciones se realizaron en el campeonato de España, competición que es el principal objetivo de la temporada de una amplia mayoría de los participantes, lo que podría condicionar los resultados. Por otra parte, la presente investigación es una investigación de corte transversal y tiene un carácter exploratorio, siendo necesario más investigaciones en el futuro que ahonden sobre los resultados encontrados en una mayor población de piragüistas y que incluyan otras variables como por ejemplo las motivacionales. Además, sería conveniente realizar dentro de este ámbito estudios longitudinales, con el fin de hondar más en el conocimiento de las variables que influyen en la aparición de burnout y sus dimensiones. 


\section{Conclusiones}

En general, los piragüistas de élite españoles muestran bajos nivel de burnout de manera global y en cada una de sus dimensiones. No obstante, los valores agotamiento físico/emocional son moderados/altos, encontrándose que la edad predice este agotamiento, siendo el agotamiento menor cuanta más edad tienen los piragüistas. Esto podría deberse a la selección natural que se produce en el deporte, quedando como practicantes los más resistentes o aquellos con más habilidad para el deporte. Es importante que aquellas personas que se encargan de la formación de los deportistas tengan en cuenta estos aspectos por la significación que tienen las variables psicológicas sobre el rendimiento del deportista, maximizando así sus posibilidades de alcanzar el éxito. El conocimiento, supervisión y control de estos elementos se ha convertido en tarea obligada para los profesionales y sobre todo para los entrenadores (Isorna et al., 2014). Sin duda, la aportación y disponibilidad de información a este nivel posee un enorme valor y constituye una herramienta clave para la optimización de los recursos y la prevención del burnout, prestando especial interés a la aparición de AFE. De este modo, tanto para los sujetos que padecen el síndrome como para los que se encuentran en riesgo de padecerlo existen en la literatura científica estrategias de intervención y programas específicos para el tratamiento del burnout y la reorientación motivacional (Sánchez-Alcaráz y GómezMármol, 2014).

\section{Referencias}

Adie, J. W., y Jowett, S. (2010). Meta-Perceptions of the Coach-Athlete Relationship, Achievement Goals, and Intrinsic Motivation Among Sport Participants. Journal of Applied Social Psychology, 40, 2750-2773. https://doi.org/10.1111/j.15591816.2010.00679.x

Appleton, P. R., y Hill, A. P. (2012). Perfectionism and athlete burnout in junior elite athletes: The mediating role of motivation regulations. Journal of Clinical Sport Psychology, 6, 129-145. https://doi.org/10.1123/jcsp.6.2.129

Arce, C., De Francisco, C., Andrade, E., Seoane, G., y Raedeke, T. (2012). Adaptation of the Athlete Burnout Questionnaire in a Spanish sample of athletes. Spanish Journal of Psychology, 5, 1529-1536. https://doi.org/10.5209/rev_SJOP.2012.v15.n3.39437

Balaguer, I., Duda, J. L., Moreno, Y., y Crespo, M. (2009). Interacciones entre las perspectivas situacionales y disposicionales de meta y el burnout psicológico de los tenistas junior de la élite internacional. Acción psicológica, 6, 63-75. http://dx.doi.org/10.5944/ap.6.2.222

Boletín Oficial del Estado (1999). Ley Orgánica 15/1999 de 13 de noviembre, de Protección de Datos de Carácter Personal. BOE de 14/12/1999.

Carfagno, D. G., y Hendrix, J. C. (2014). Overtraining syndrome in the athlete: Current clinical practice. Current sports medicine reports, 13, 45-51. https://insights.ovid.com/crossref?an=00149619-201401000-00013

De Francisco, C., Garcés de los Fayos, E., y Arce, C. (2014). Burnout en deportistas: Prevalencia del síndrome a través de dos medidas. Cuadernos de Psicología del Deporte, 14, 29-38.

Feigley, D. A. (1984). Psychological burnout in high-level athletes. The Physician and Sports Medicine, 12, 109-119. https://doi.org/10.1080/00913847.1984.11701971

(c) Psy, Soc, \& Educ, 2019, Vol. 11(1) 
Garcés de Los Fayos, E. (1999). Nivel de burnout y rendimiento deportivo. En E. Cantón (Ed.), Motivación y su Aplicación al Deporte (pp. 91-102). Valencia, España: Promolibro.

Garcés de Los Fayos, E., y Vives, L. (2002). Variables motivacionales y emocionales implicadas en el síndrome de burnout en el contexto deportivo. Revista electrónica de motivación y emoción, 5(11-12). Consultado el 25 febrero de 2015 en http://reme.uji.es/articulos/agarce2110312102/texto.html

Garcés de Los Fayos, E., y Cantón, E. (2003). El cese de la motivación: el síndrome del burnout en deportistas. Revista de Psicología del Deporte, 7, 151-160.

Garcés de Los Fayos, E., y Medina, G. (2002). Principios básicos a aplicar en el desarrollo de programas de intervención y prevención en deportistas con el síndrome de burnout. Propuestas desde una perspectiva transnacional. Revista de Psicología del Deporte, 11, 259-267.

Garcés de Los Fayos, E.J., Olmedilla, A., y Jara, P. (2006). Psicología y deporte. Murcia, España: Diego Marín.

García-Jarillo, M., De Francisco, C., y Garcés de Los Fayos, E. J. (2016). El síndrome de burnout en deportistas: estudio piloto sobre la percepción del psicólogo deportivo a través del método Delphi. Cuadernos de Psicología del Deporte, 16, 243-250.

García-Parra, N., González, J., y Garcés de los Fayos, E. J. (2016). Estado actual del estudio del síndrome de burnout en el deporte. Cuadernos de Psicología del Deporte, 16, 21-28. http://scielo.isciii.es/pdf/cpd/v16n2/psicologia2.pdf

Gómez, M., Granero, A., e Isorna, M. (2013). Análisis de los factores psicológicos que afectan a los piragüistas en el alto rendimiento. Revista Iberoamericana de Diagnóstico y Evaluación Psicológica, 35, 57-76. http://www.redalyc.org/pdf/4596/459645435004.pdf González, J., y Ortín, F.J. (2010). Indicadores de rendimiento y cooperación deportiva. Cuadernos de Psicología del Deporte, 10, 57-61.

Gould, D., Dieffenbach, K., y Moffett, A. (2002). Psychological characteristics and their development in Olympic champions. Journal of Applied Sport Psychology, 14, 172-204. https://doi.org/10.1080/10413200290103482.

Gustafsson, H., Kenttä, G., y Hassmeén, P. (2011). Athlete burnout: An integrated model and future research directions. International Review of Sport and Exercise Psychology, 4, 324. https://doi.org/10.1080/1750984X.2010.541927

Gustafsson, H., Kenttä, G., Hassmeén, P., y Lundqvist, C. (2007). Prevalence of burnout in adolescent competitive athletes. The Sport Psychologist, 21, 21-37. https://doi.org/10.1123/tsp.21.1.21

Harris, B., y Smith, M. (2009). The influence of motivational climate and goal orientation on burnout: An exploratory analysis among Division I collegiate student-athletes. Athletic Insight: The Online Journal of Sport Psychology, 11, 39-56.

Hill, A. P., Hall, H. K., Appleton, P. R., y Murray, J. J. (2010). Perfectionism and burnout in canoe polo and kayak slalom athletes: The mediating influence of validation and growth-seeking. The Sport Psychologist, 24, 16-34.

Hoar, S. D., Kowalski, K. C., Gaudreau, P., y Crocker, P. R. E. (2006). A review of coping in sport. En S. Hanton y S. D. Mellalieu (Eds.), Literature reviews in sport psychology (pp. 47-90). New York, Estados Unidos: Nova Science Publishers. 
Isorna, M., Navarro, F., y Alacid, F. (2014). Planificación deportiva aplicada al piragüismo. En M. Isorna, F. Alacid y J. Mangas, Entrenamiento en piragüismo de Aguas Tranquilas (pp.13-76). A Coruña: 2.0 Editora.

Lemyre, P. N., Hall, H., y Roberts, G. (2008). A social cognitive approach to burnout in athletes. Scandinavian Journal of Medicine \& Science in Sport, 18, 221-234. https://onlinelibrary.wiley.com/doi/abs/10.1111/j.1600-0838.2007.00671.x Lenz, J. (2008). Performance and Theory of Canoe Training. Leipzig, Alemania: Pan-Hellenic Kajak and Canoe Trainers Association.

López, M. G., Gallegos, A. G., y Isorna, M. (2013). Análisis de los factores psicológicos que afectan a los piragüistas en el alto rendimiento. Revista iberoamericana de diagnóstico y evaluación psicológica, 1, 57-76.

Medina, G., y García-Ucha, F. (2002). Burnout, locus de control y deportistas de alto rendimiento. Cuadernos de Psicología del Deporte, 2, 29-42.

Nitsch, J., Neumaier, A., Marées, H., y Mester, J. (2002). Entrenamiento de la técnica: contribuciones para un enfoque interdisciplinario. Barcelona: Editorial paidotribo.

Pedrosa, I., y García-Cueto, E. (2014). Estudio del síndrome de burnout en deportistas: prevalencia y relación con el esquema corporal. Universitas Psychologica, 13, 135-143. http://www.scielo.org.co/pdf/rups/v13n1/v13n1a12.pdf

Pérez-Rubio, C., González, J., y Garcés de los Fayos, E. J. (2017). Personalidad y burnout en jugadores profesionales de e-sports. Cuadernos de Psicología del Deporte, 17, 41-50. http://scielo.isciii.es/pdf/cpd/v17n1/original5.pdf

Price, M., y Weiss, M. (2000). Relationships among coach burnout, coach behaviors, and athletes psychological responses. The Sport Psychologist, 14, 391-409. https://doi.org/10.1123/tsp.14.4.391

Raedeke, T. D. (1997). Is burnout more than just stress? A sport commitment perspective. Journal of Sport and Exercise Psychology, 19, 396-417. https://doi.org/10.1123/jsep.19.4.396

Raedeke, T.D., y Smith, A.L. (2001). Development and preliminary validation of an athlete burnout measure. Journal of Sport \& Exercise Psychology, 23, 281-306. https://doi.org/10.1123/jsep.23.4.281

Rascado, S. M., Rial, A., Isorna, M., y Fernández, D. A. (2014). Niveles de rendimiento y factores psicológicos en deportistas en formación. Reflexiones para entender la exigencia psicológica del alto rendimiento. Revista Iberoamericana de Psicología del Ejercicio y el Deporte, 9, 373-392.

Reynaga, P., y Pando, M. (2005). Relación del síndrome de agotamiento crónico (Burnout) con el trastorno psicológico potencial en jóvenes deportistas. Investigación en Salud, 7, 153187.

Rial, A., Marsillas, S., Isorna, M., y Louro, A. (2013). Recomendaciones para el Apoyo psicológico de un Jóvenes Deportistas en los Centros de Alto Rendimiento. Consejos para el apoyo psicológico a los atletas jóvenes en centros de alto rendimiento. Revista Internacional de Ciencias del Deporte, 9, 252-268. http://dx.doi.org/10.5232/ricyde2013.03304 
Saies, E., Arribas, S., Cecchini, J. A., Luis-De-Cos, I., y Otaeg, O. (2014). Diferencias en orientación de meta, motivación autodeterminada, inteligencia emocional y satisfacción con los resultados deportivos entre piragüistas expertos y novatos. Cuadernos de Psicología del Deporte, 14, 21-30.

Sánchez-Alcaraz, B., y Gómez-Mármol, A. (2014). Prevalencia del síndrome de Burnout en tenistas según su orientación motivacional. Revista Iberoamericana de Psicología del Ejercicio y el Deporte, 9, 111-122.

Tutte, V., Blasco, T., y Cruz, J. (2006). Evolución de los índices de burnout en un equipo femenino de baloncesto. Cuadernos de Psicología del Deporte, 6, 21-35.

Tutte, V., y Reche, C. (2016). urnout, resiliencia y optimismo en el hockey sobre hierba femenino. Cuadernos de Psicología del Deporte, 16, 73-78.

Vives, L., y Garcés de los Fayos, E. (2004). Incidencia del síndrome de burnout en el perfil cognitivo en jóvenes deportistas de alto rendimiento. Cuadernos de Psicología del Deporte, 4, 29-43. 